\title{
Long COVID from rheumatology perspective - a narrative review
}

\author{
Hem Raj Sapkota ${ }^{1}\left[\right.$. Arvind Nune ${ }^{2}(\mathbb{D}$
}

Received: 16 September 2021 / Revised: 7 November 2021 / Accepted: 21 November 2021 / Published online: 30 November 2021

(c) International League of Associations for Rheumatology (ILAR) 2021

\begin{abstract}
Long-term sequel of acute COVID-19, commonly referred to as long COVID, has affected millions of patients worldwide. Long COVID patients display persistent or relapsing and remitting symptoms that include fatigue, breathlessness, cough, myalgia, arthralgia, sleep disturbance, cognitive impairment and skin rashes. Due to the shared clinical features, laboratory and imaging findings, long COVID could mimic rheumatic disease posing a diagnostic challenge. Our comprehensive literature review will help rheumatologist to be aware of long COVID manifestations and differentiating features from rheumatic diseases to ensure a timely and correct diagnosis is reached.
\end{abstract}

Keywords Long COVID $\cdot$ Post-COVID-19 syndrome $\cdot$ Post COVID-19 condition · Rheumatology $\cdot$ Systemic autoimmune rheumatic diseases

\section{Introduction}

Post-viral sequelae are reported following many bacterial and viral infections. Hickie et al. described that $12 \%$ of patients presented with disabling fatigue, musculoskeletal pain, cognitive difficulties and mood disturbance 6 months after the diagnosis of a viral illness [1]. Reactive arthritis following gastrointestinal infections and sexually transmitted diseases (STD) is a well-known phenomenon. Patients recovering from acute-COVID-19 have also demonstrated a range of symptoms that have lasted for several months after recovery. This has been widely known as "long COVID". Many studies have evaluated long COVID symptoms in hospitalised patients, which inevitably meant that only the more severe end of the spectrum was included.

An estimated incidence of long COVID following acute COVID-19 has been reported to be up to 30\% [2]. As per the Office for National Statistics (ONS), 1.5 million people living in private households were experiencing self-reported

Hem Raj Sapkota

Hem.sapkota@nhs.net

Arvind Nune

arvind.nune@nhs.net

1 The Royal Wolverhampton Hospital NHS Trust, Wolverhampton WV10 0QP, UK

2 Southport and Ormskirk NHS Trust, Southport PR8 6PN, UK long COVID symptoms as of 1 August 2021, in the UK alone [3]. Of these, 384,000 had or were suspected of having COVID-19 a year ago. This is defined as symptoms persisting for more than 4 weeks after the first suspected acute COVID-19 that cannot be attributed to an alternative diagnosis [3]. As of the same date, there had been 5.9 million confirmed cases of COVID-19 in the UK. Community cases were also not accounted for due to the lack of widespread testing during the first wave of the pandemic [4]. Along with the rising incidence of acute COVID-19 cases, the proportion of patients with long COVID will inevitably increase. Collectively, this highlights the extent of the burden that long COVID has posed to the current healthcare system in the UK and across the globe.

The most commonly reported long COVID symptoms include breathlessness, cough, fatigue, arthralgia, sleep disturbance and myalgia. Systemic autoimmune rheumatic diseases (SARDs), including rheumatoid arthritis (RA), connective tissue disease (CTD), idiopathic inflammatory myositis (IIM) and fibromyalgia (FM) and chronic fatigue syndrome (CFS), share similar symptoms with long COVID. Patients with a history of rheumatic diseases and a previous diagnosis of COVID-19 who have developed persistent joint or muscle symptoms pose a significant challenge to clinicians. Such patients may be experiencing long COVID or a flare-up of their pre-existing rheumatic disease.

This article provides a comprehensive literature review of long COVID symptoms and highlights the differentiating 
features between long COVID and SARD. We also provided an easy-to-follow management algorithm when facing a patient with shared clinical features.

\section{Long COVID — definition}

Long COVID is defined as a long-term sequel of a SARSCoV-2 infection, presenting as continuous or relapsing and remitting symptoms several weeks after microbiological recovery, as evidenced by negative SARS-CoV-2 PCR. In most patients, there is also evidence of radiological and biochemical recovery. Hence, long COVID represents a time lag between microbiological and clinical recovery. Some patients with long COVID may still present with haematological/biochemical and imaging abnormalities [5, 6].

No clear consensus had been agreed to define long COVID, with several inconsistencies in the proposed definitions [7]. Initial reports defined long COVID as the persistence of symptoms 4 weeks post-recovery from an acute infection [8]. National Institute for Health and Care Excellence (NICE) in the UK has named long COVID as "post-COVID-19 syndrome" [9]. The NICE defines the post-COVID-19 syndrome as symptoms that arise during or following an infection consistent with COVID-19. These symptoms persist for more than 12 weeks and cannot be attributable to an alternative diagnosis. This has yielded some clarity regarding the definition of long COVID in the UK. In October 2021, World Health Organisation (WHO) has defined long COVID as "post COVID-19 condition" which occurs in individuals with a history of probable or confirmed SARS-CoV-2 infection, usually 3 months from the onset of COVID-19 with symptoms that last for at least 2 months, which cannot be explained by an alternative diagnosis [10]. It is also referred to as post-acute, chronic or long haul COVID-19 [8]. For the purpose of this review, we have used the term long COVID due to its wide usage.

Besides the limited clarity of the definition of long COVID, the lack of mass testing across the globe during the first wave of the COVID-19 pandemic resulted in inconsistent available data. Additionally, the incidence of asymptomatic disease should also be considered, as this poses a diagnostic challenge if they develop symptoms indicative of long COVID. COVID-19 antibody testing helps to identify past infection, but not every patient may seroconvert, and the longevity of the antibodies is poorly understood $[11,12]$.

The diagnosis of COVID-19, and hence long COVID, can be made based on a patient's symptoms retrospectively with or without a positive SARS-CoV-2 test (PCR, antigen, or antibody). However, a retrospective diagnosis of COVID-19, and long COVID, has several limitations.

\section{Symptoms of long COVID: literature search}

It is not well known why some patients develop long COVID [13]. The current evidence substantiates that long COVID risk is not linked to the severity of the acute COVID-19 infection [3]. Although women present with a survival benefit following an acute COVID-19 infection, they are more likely to suffer from fatigue after recovery and to develop long COVID [14, 15].

To understand the full spectrum of long COVID symptoms, we performed a comprehensive literature search using PubMed published between 01 Jul 2020 to 30 Jun 2021. The keywords implemented in the search strategy were "long COVID" or "post-COVID-19 syndrome" or "long haul COVID-19" or "chronic COVID-19" or "postacute COVID-19" to ensure the full scope of literature was included. Citations published in any non-English language were excluded to prevent the misinterpretation of findings during the translation. The search strategy yielded 4913 articles. After screening for relevance, 4726 articles were excluded, which did not meet our study objectives. We further reviewed the remaining 187 full-text articles. This resulted in the exclusion of an additional 144 citations. We also excluded studies on children with long COVID, patients who had recovered and the studies which did not include long COVID symptoms as their primary study objective. The remaining 43 research articles were included in our literature review (Fig. 1).

Table 1 summarizes the long COVID symptoms reported in the literature identified in our search strategy. Fatigue, dyspnoea, cough, chest pain, dysgeusia/anosmia, myalgia, diarrhoea, hair loss and myalgia were the most frequently reported symptoms. A recently published meta-analysis of long COVID corroborated the following as the most prevalent symptoms of long COVID: fatigue (58\%), headache (44\%), attention disorder (27\%), hair loss (25\%) and dyspnoea (24\%) [16]. As per ONS in the UK, fatigue (58\%) was also the most common self-reported long COVID-symptom followed by breathlessness (42\%), muscle ache (32\%) and difficulty in concentration (31\%) [3].

\section{Long COVID and rheumatology}

Long COVID remains a diagnosis of exclusion, and patients should be appropriately evaluated before reaching a final diagnosis. Before confirming long COVID, SARD should be considered in a differential diagnosis as manifestations of several rheumatic diseases may mimic long COVID. The current literature consists of numerous observational studies and review articles that have outlined 
Records identified through database search (4913)

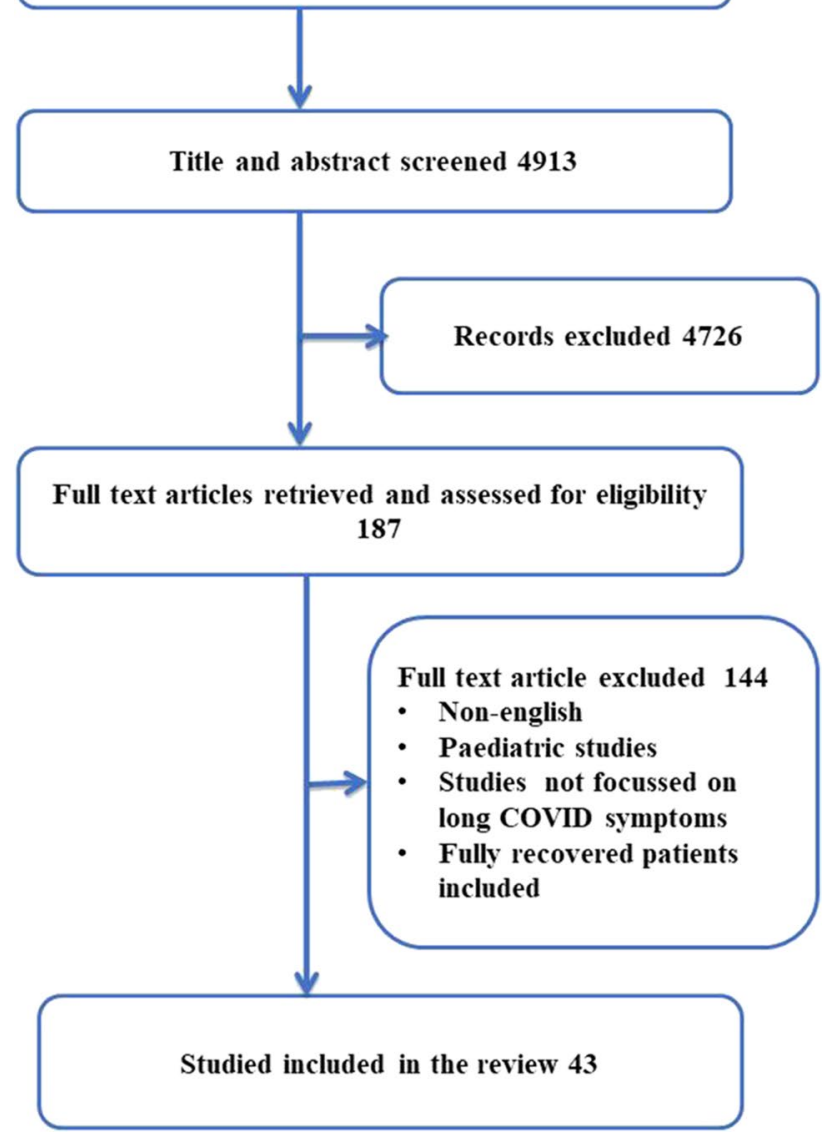

Fig. 1 Flowchart study selection of long COVID

the long-term consequences of long COVID [24]. Here, we provide an overview of overlapping features between these two conditions and explain the possibility of a potential misdiagnosis if long COVID was not considered during the work-up of SARD in the post-COVID-19 era.

\section{Blood abnormalities and autoantibodies}

Vlachoyiannopoulos et al. reported incidence of autoantibodies related to SARD in severe acute COVID19 cases [25]. Out of a total of 29 patients studied, 69\% of patients were found to have one of the following autoantibodies suggesting post-COVID-19 autoimmune reaction: antinuclear antibody (ANA), antineutrophil cytoplasmic antibodies (ANCA), anti-cardiolipin (aCL), anti-beta-2-glycoprotein-1 (anti- $\beta 2 \mathrm{GP} 1$ ) and anti-cyclic citrullinated peptide (anti-CCP). The most commonly observed autoantibodies in COVID-19 patients were ANA, $\mathrm{aCL}$ and anti- $\beta 2 \mathrm{GP} 1$. There were also four ANCA-positive patients who tested negative for MPO (myeloperoxidase) and PR3 (Proteinase 3). Pre-COVID immunology status was not available in this study. The titre of the autoantibodies when present was generally low; for example, ANA $\leq 1 / 320$. A further study by Lerma et al. noted similar findings in $25 \%$ of their patients who were ANA positive as per manufacturerrecommended threshold, and RNP was the most commonly positive extractable nuclear antigen (ENA) [26]. However, the titre was low and deemed not clinically significant. The low titre of ANA or other autoantibodies could result from transient autoreactive B cell and plasma cell activation [27]. A recent case series by Derken et al. evaluated the serology of 61 patients 5 weeks after COVID-19 infection but found no increased incidence of anti-CCP positivity [28].

The duration of positive auto-antibodies presence following COVID-19 illness is poorly understood. The pattern of autoantibodies found following infection was seemed to be due to a reactive phenomenon; hence, we can extrapolate data from literature studies concerning other viral illnesses. This would suggest that autoantibodies are short-lived and fade away eventually in COVID-19 patients [29]. It is unlikely that patients develop strongly positive autoantibodies in response to acute SARS-CoV-2 infection. Therefore, the presence of strongly positive autoantibody would favour the diagnosis of SARD.

Lymphopenia is a characteristic feature of CTD; yet it is also observed in patients with long COVID. In a study of 384 patients, the blood abnormalities such as D-dimer, C-reactive protein (CRP), ferritin noted prior to hospital discharge had returned to normal in many patients at a median of 54 days follow-up [6]. Of those with abnormal pre-discharge blood results, $7.3 \%$ of 247 patients had persistent lymphopenia, whilst $9.5 \%$ of 190 patients had elevated CRP at the follow-up. This study emphasises that long COVID should be included in differential diagnoses in a patient with lymphopenia and raised inflammatory markers, often encountered in SARD in an appropriate clinical context. Another study by Raman et al. reported magnetic resonance imaging (MRI) changes in post-COVID patients' lungs, heart, kidneys and liver who presented with predominant fatigue and breathlessness [30]. However, the lack of pre-COVID imaging is a limitation of this study. Interestingly, the abnormalities detected on the MRI correlated with inflammatory markers such as CRP. This suggests a potential relationship between chronic inflammation and organ damage among patients with long COVID. This further gives rise to the diagnostic challenges caused by long COVID, in patients with no previous imaging or blood investigations, particularly in nations with a lower socioeconomic status.

Elevated IL-6 is associated with persistent pulmonary lesions in COVID-19 patients following their acute recovery [31]. Also, patients with evidence of pulmonary fibrosis after recovering from acute COVID-19 had a higher level of systemic inflammation at admission (ESR, CRP and D-dimer) and bone marrow suppression as evidenced by 


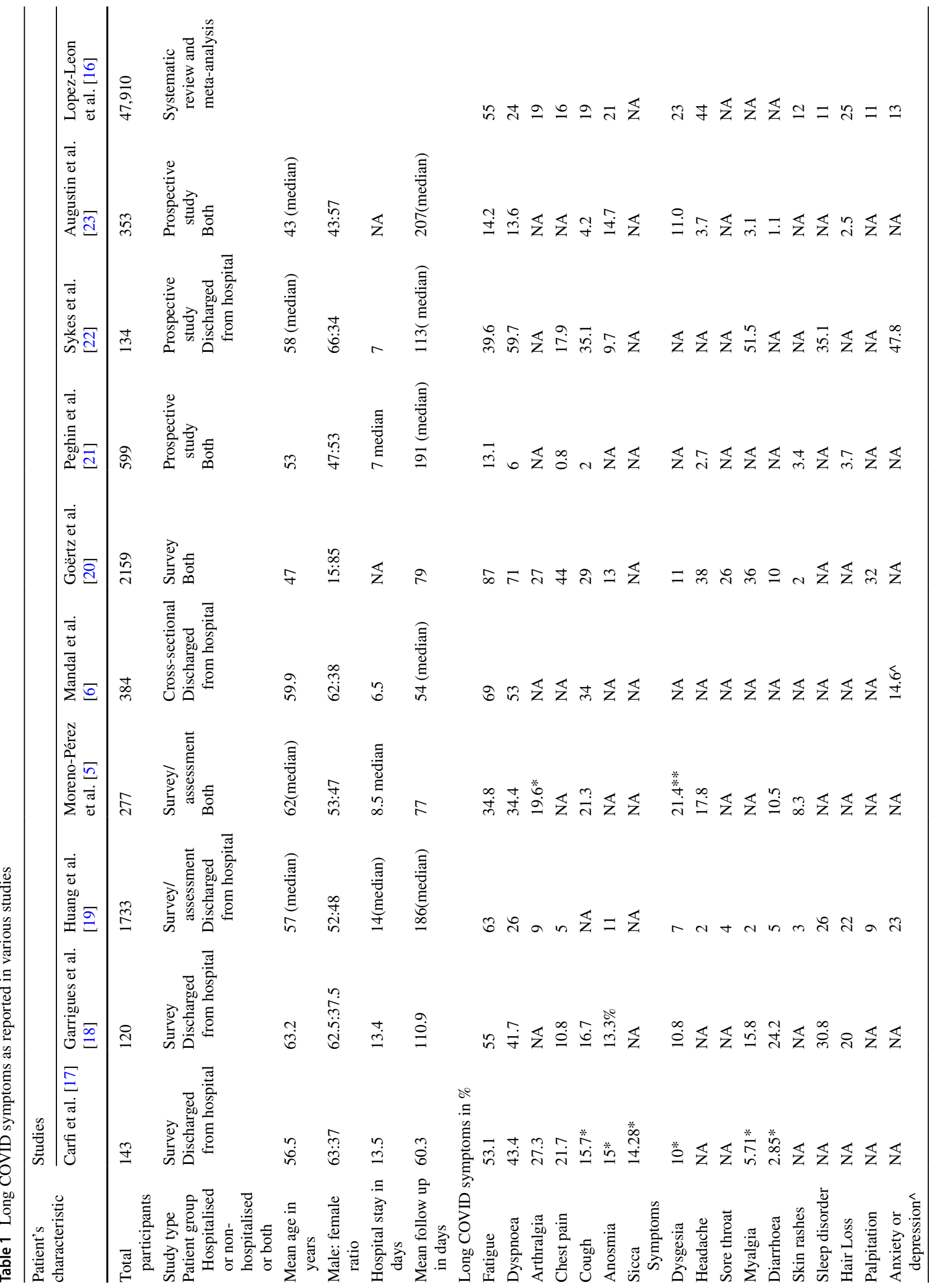


thrombocytopenia, leukopenia and low haemoglobin [32]. These parameters could be used as a potential biomarker for long COVID. However, such findings have not been consistently replicated in other studies. Moreno-Perez et al., in their study, found that baseline clinical features such as CRP, D-dimer were not associated to predict long COVID [5]. Large prospective studies are needed to define the biomarkers' association with chronic inflammation in patients with long COVID.

\section{Arthralgia/inflammatory arthritis}

Respiratory infections can cause an onset of new rheumatoid arthritis and a flare-up of an existing RA [33]. Reactive arthritis typically follows urethral and GI infections and is also known to occur after viral infections, such as dengue, chikungunya, parvovirus B19 and rubella. COVID-19 has also been found to cause reactive arthritis and new-onset inflammatory arthritis typically occurring within a month after its diagnosis $[34,35]$. Several cases of reactive arthritis have been reported so far after post-COVID-19 infection [36]. They share similar clinical features such as the onset of reactive arthritis occurring a week after resolution of COVID-19 symptoms, male predominance with an age range between 40 and 70, lower extremities involvement and elevated CRP [36].

Arthralgia has been a widely reported symptom of long COVID. Peterson et al. highlighted in their study that arthralgia was the most common long COVID symptom [37]. A systematic review further substantiated these findings that an estimated one in five patients with long COVID suffers from arthralgia [16].

In a Dutch study of five patients who presented with inflammatory arthritis 6.6 weeks post COVID-19 infection, two patients had strongly positive and another patient had weakly positive anti-CCP antibodies [28]. However, serology status before the presentation was not available. Based on clinical phenotype and autoantibodies characterisation, Derksen et al. proposed that the new onset of RA post-COVID-19 could be coincidence rather than causality. Interestingly, another case report of a middle-aged woman who developed a seropositive RA with elevated CRP and erythrocyte sedimentation rate (ESR) 2 weeks post-hospital discharge after a COVID-19 infection had previously tested negative for RF and anti-CCP. Upon re-testing, this patient had developed positive RF $131 \mathrm{IU} / \mathrm{mL}(<10 \mathrm{IU} / \mathrm{mL})$, positive anti-CCP 35 $\mathrm{U} / \mathrm{mL}(<20 \mathrm{U} / \mathrm{mL})$, who also had confirmed synovitis in both wrist joints on an ultrasound scan (US). This patient needed methotrexate (MTX) to manage his active RA. Seroconversion with new onset of RA, in this case, suggests COVID-19 infection as a trigger for autoimmunity [38].

Well-designed studies are warranted to confirm or refute their association with the onset of RA. Nevertheless, it is 
recommended that if a patient develops clinical features of inflammatory arthritis following a COVID-19 infection, the diagnostic work-up should be similar to a patient with a newonset RA in an infection naive patient.

\section{Myalgia/idiopathic inflammatory myositis}

Myalgia is a prevalent symptom during the acute COVID19 infection. In one study, almost half of their patients presented with myalgia and 10 out of 37 patients had raised CK [39]. Evidence of myositis on EMG, MRI and muscle biopsy following COVID-19 has also been reported, but there is no evidence to confirm the presence of myositis specific antibodies [40-42]. Cases of rhabdomyolysis due to acute COVID-19 have also been reported [43]. Muscle involvement in long COVID has also been reported as seen in this case of myocarditis which occurred about a month after recovering from acute COVID-19 [44].

Myalgia is also a common symptom in patients with long COVID, SARD and FM. As many as one-third of long COVID patients suffer from it [3]. Unlike its presentation in patients with long COVID, myalgia in SARD, such as SLE, SS, scleroderma and mixed CTD, is commonly associated with other manifestations, such as Raynaud's, skin rashes and strongly positive autoantibodies. For example, a patient with generalised pain with malar rash and mouth ulcers raises the possibility of SLE. The autoantibodies commonly present in SARD include Ro and La, anti-centromere, anti-Scl-70 and anti-ribonucleoprotein (RNP), anti-smith (anti-sm) and anti-double-stranded deoxyribonucleic acid (ds-DNA), which are disease-specific. Clinically significant titres of these antibodies are less likely to be found in patients with long COVID [26]. If present, a weakly positive ANA is a false positive result with no clinical significance especially in the absence of CTD symptoms. Myalgia in patients with FM is not commonly associated with muscle weakness or raised CK, contrary to high CK in IIM.

\section{Breathlessness}

Breathlessness is one of the common respiratory symptoms of long COVID, with a prevalence of about $37 \%$, which is also frequently present in patients with SARD [45]. $\mathrm{Wu}$ et al. demonstrated that persistent physiological and radiological abnormalities were apparent in $88 \%$ and $24 \%$ of patients post-COVID-19 at 12 months after their hospital discharge, respectively [46]. Breathlessness in patients with long COVID has also been attributed to immune-mediated disruption to the autonomic nervous system, resulting in transient or long-term orthostatic intolerance syndromes without any structural lung abnormality, or as a result of pulmonary fibrosis [47]. Therefore, a new event or worsening of the existing interstitial lung disease (ILD) in a patient with SARD could cause a dilemma regarding the underlying aetiology in a patient recovering from COVID-19. A review of previous imaging, if available, will help to distinguish between SARD and SARS-CoV-2-related lung changes [47]. British Thoracic Society (BTS) guidelines recommended a repeat CXR at 12 weeks to evaluate ILD for patients with COVID-19 pneumonia [48]. Follow-up imaging with highresolution CT (HRCT) should be considered in patients with persistent imaging changes, if they are symptomatic. The resolution of lung changes could indicate that initial lung changes are perhaps due to COVID-19 infection.

The most common CT manifestations of COVID-19 pulmonary involvement are bilateral, peripheral/subpleural, posterior ground-glass opacities with or without consolidations, with a predisposition to the lower lung zones [49]. In most patients, lung imaging changes persisted at hospital discharge [50]. These changes have resolved in many patients over the next few days, but in a small proportion of patients, lung changes had evolved into pulmonary fibrosis [51]. In a study by Mandal et al., 9\% (23/244) of patients had a significant deterioration in their CXR findings at the median follow-up period of 54 days [6, 52]. In another study, $42 \%$ of patients demonstrated residual lung changes at the 3-month follow-up between the first and second CT scans [53]. Imaging findings often observed in systemic sclerosis are either non-specific interstitial pneumonia (NSIP) or usual interstitial pneumonia (UIP), which manifest as ground-glass opacities, and reticulation respectively [54]. These patterns are also seen in patients with COVID-19 pneumonia. Therefore, a thorough review of the radiological imaging preceding COVID-19 infection and evaluating the clinical features and autoantibodies is vital to establish this distinction.

Similarly, the CT chest findings in patients with IIM, anti-synthetase syndrome, systemic sclerosis and mixed CTD are associated with ILD, which can be mistaken for COVID-19 pneumonia as all these conditions could present with ground-glass opacities. Although a rare presentation, in an estimated $1-8 \%$ of cases, pleural effusion may also be observed in COVID-19 pneumonia [55]. IIM patients may also have pleural effusion due to underlying malignancy. When the pleural effusion is present in patients with ILD in IIM, it may enable the differentiation with COVID-19 pneumonia. Thoracentesis may help to differentiate these conditions when the diagnosis is ambiguous. Clinical features, including Gottron's papules, heliotrope rash in dermatomyositis (DM); evidence of myositis on EMG and histopathology findings in PM; fever, mechanics hands and rapidly progressive ILD in anti-synthetase syndrome, coupled with disease-specific autoantibodies will enable to reach the correct diagnosis. Ground-glass changes in long COVID frequently presented in the dependent area of the lung which can be misinterpreted as atelectasis [53]. As a result, it is pertinent that clinicians are aware of various 
imaging findings and differentiating features which may present in patients with long COVID, and SARD will help with an accurate diagnosis and prevents any management delay. A lung biopsy may also be considered to ascertain a definite aetiology of pulmonary involvement if required. Therefore, a multi-disciplinary team (MDT) approach involving radiologists, respiratory clinicians and histopathologist is essential to manage these patients when in doubt.

\section{Dermatological manifestation}

A variety of dermatological manifestations following COVID-19 infection are widely reported. One systematic review conducted after the first wave of the pandemic described 27 different skin lesions in patients associated with COVID-19 infection. These included pernios (16.56\%), morbilliform exanthema (13.50\%), vesicular eruption $(13.19 \%)$, urticaria $(9.82 \%)$, erythematous exanthema (7.98\%), papulosquamous-eruption (5.52), retiform purpura (3.68) and livedo-reticularis lesions (2.76\%) [56].

Patients developing new skin lesions following full recovery from acute COVID-19 infection have also been recognised. The International Dermatology Registry data informed that skin symptoms in long COVID are not uncommon and could last up to 150 days [57]. As per the registry data, pernios lesions and livedo reticularis persist for the longest duration. Other lesions in long COVID are morbilliform, urticarial, macular erythema, vesicular, papulosquamous and retiform-purpura.

Many skin lesions seen in long COVID are also commonly seen in SARD. Pernio lesions are seen as red or purplish patches in toes and are usually called "COVID toes", which are more commonly seen in young patients with a relatively mild form of COVID-19 infection. They resemble chilblains, a common presenting feature in patients with CTD such as SLE. A case report also described a palmar/plantar petechial rash several weeks after COVID-19 infection due to small-vessel vasculitis [58]. These skin lesions could be mistaken for the manifestation of SARD, and the clinician must remain vigilant. Skin lesions may result from microvascular changes in hands and feet similar to CTDs, and therefore, nail fold capillaroscopy (NFC) could potentially be used to differentiate this [59]. It is not known whether long COVID patients develop nail fold changes seen in secondary Raynaud's such as enlargement of capillary loops, loss of capillaries and capillary tortuosity. The aforementioned NFC changes are often helpful to differentiate Raynaud's due to SARD from primary Raynaud's [60]. A similar treatment algorithm, used in digital ischemic in CTD, has been proposed to treat prolonged refractory COVID toes [59].

\section{Fatigue}

Fatigue, a debilitating symptom of long COVID, is also shared by several rheumatic conditions. CFS, FM and SARD frequently cause fatigue and widespread pain. FM and CFS share a common pathophysiological process (central sensitization) and can be triggered by stressors (physical, mental, emotional, financial) affecting at-risk patients [61]. Although evidence is still emerging, fatigue, a common symptom of long COVID, may belong to the same spectrum of the FM/CFS [62]. A systematic review recently compared the clinical symptoms of long COVID to myalgic encephalomyelitis (ME)/CFS [63]. Out of 29 listed ME/CFS symptoms, 25 were reported by at least one of the 21 studies included in the review. All three major diagnostic criteria of CFS/ME, namely fatigue, post-exertional malaise and reduced daily activity, were reported by patients in many studies. Brain fog represents one of the most common neurological cognitive symptoms of long COVID reported in a study by Graham et al. [64]. In this study, among 100 patients, the incidence of brain fog was identified in $81 \%$ of patients, 4.7 months after the onset of symptom followed by headache in $68 \%$ of the participants. In another study by Ursini et al., 30.7\% (189 out of 616 patients) fulfilled the American College of Rheumatology (ACR) classification criteria for fibromyalgia $6 \pm 3$ months after the diagnosis of acute COVID-19 [65]. Obesity and male gender were risk factors of developing FM in this study. Thus, fatigue in a patient with long COVID may be a manifestation of FM and would need an appropriate assessment.

Even though more research is needed to outline the management plan, similar treatment modalities used in ME/ CFS may be helpful to support the recovery of patients with long COVID. Holistic measures such as graded exercise/ pacing, mindfulness, coping mechanisms and sleep hygiene should be part of the management for these patients. This management strategy is further supported as long COVID and ME/CFS may share similar pathophysiology, particularly with post-viral chronic fatigue syndromes, such as dysregulated immune system and hyper-inflammatory state [66]. Several other studies have noted fewer patients presenting with systemic exertion intolerance disease within 6 months after COVID-19 infection [67]. Thus, it is fair to assume that the features of post-acute COVID-19 syndrome overlap with the clinical features of ME/CFS.

\section{Discussion}

Besides the symptoms discussed above, many additional symptoms relevant to rheumatology have been reported following the post-COVID-19 infection. One of these 
symptoms frequently reported includes back pain, with more than $19 \%$ of the patient in a cohort reported increased back pain [68]. The back pain appeared to be mechanical, suggesting a relationship between home working, weight gain and the reduced physical activity that arose during the lockdown, rather than an increased incidence of inflammatory back pain. Headache and sicca symptoms have also been frequently reported among long COVID symptoms.

Both SARD and COVID-19 share a similar pathophysiological basis caused by exaggerated immune response characterised by cytokine stimulation and hyperactivity [69]. Both innate and adaptive immune systems play a key role leading to macrophage activation and cytokine storm. Molecular mimicry and crossreactivity are thought to trigger an immune response in patients with COVID-19 in developing SARD. Vojdani et al. described cross-reactivity of human monoclonal autoantibody against SARS COV-2 spike protein, nucleoproteins and peptides [70]. Due to shared pathophysiology, immunosuppressive drugs such as corticosteroids, Janus kinase inhibitors, IL-1 and IL-6 blockers have been successfully used to treat inflammatory complications of COVID-19.

The most common symptoms of long COVID, such as fatigue, breathlessness, cough and arthralgia, are subjective. Only anosmia, sicca symptoms and skin rashes could be objectively assessed, but these symptoms' incidence was either low or not widely reported. As discussed in this manuscript, there has been evidence of abnormalities in blood indices and persistent inflammation in various organs. However, there is not ample data to support the view that this is common occurrence in patients recovering from acute COVID-19. Therefore, we can assume that other factors such as the sequel of critical illness and hospital deconditioning may play a role in developing long COVID symptoms [71, 72]. Central, peripheral autoimmune nervous system-induced proinflammatory response, psychosocial factors and delayed recovery in an at-risk patient with comorbidity may also play a role [73].

It is worth noting that there are also emerging cases of rheumatic-immune mediated inflammatory disease (R-IMD) following SARS-CoV-2 vaccinations reported recently [74]. There was no plausible trigger for R-IMD flares or New-Onset Disease in 27 subjects following SARS-CoV vaccinations. However, tests for acute COVID-19 were not carried out. More recently, a flare-up of well-controlled RA has been published following the administration of the SARS-CoV-2vaccination [75]. In another case, a young male patient exhibited several characteristic features for SLE, including inflammatory arthritis, myalgia and strongly positive immunology (ANA
1:2560, ds DNA > 379 units/ml) (normal range 0-1.9) after SARS-CoV-2 vaccination which might have been a new onset of SLE after the vaccination in a genetically predisposed individual or merely a co-incidence [76]. This patient was tested negative for SARS-CoV-2 but COVID19 antibodies were not checked; hence, we cannot exclude previous COVID-19 infection or asymptomatic disease.

Long COVID will remain a diagnostic challenge, given the similarities it shares with SARD, including elevated inflammation markers, presence of autoantibodies and emerging evidence of organ-specific inflammatory changes on imaging $[6,30]$. A careful history, physical examination and appropriate investigations will enable clinicians to differentiate these conditions. However, due to the multi-organ involvement, clinicians should be educated about long COVID. The management of long COVID should involve a multidisciplinary approach due to the complexity of this clinical syndrome. Additionally, we recommend that clinicians should also be part of the MDT in long COVID clinics, including occupational therapists, physiotherapists, clinical psychologists and rehabilitation clinicians, to provide a holistic approach to the management.

Therefore, we have outlined a pragmatic algorithm (Fig. 2) to differentiate long COVID from SARD based on our experience of managing SARD and the available evidence of long COVID. We hope that our algorithm will be a useful clinical tool for the clinicians to remind the shared clinical presentations of long COVID and SARD. The algorithm would also help triage and streamline rheumatology referrals, which are already inundated with the increasing workload in post COVID era.

We feel that the management of long COVID should start at the time of acute-COVID-19 diagnosis, and patients and their carers should receive the appropriate education and help if required. Given the debilitating effects of long COVID, patients with COVID-19 should be assessed at the appropriate interval following their initial infection for long COVID as per country-specific guidelines [9]. Services directed to provide appropriate management to long COVID patients' have attracted some criticism, and many patients felt that they were "unsupported" [13]. To address this, long-COVID clinics in the UK and the other parts of the world have been already set up; however, patients' outcomes and satisfaction inform us how effective this initiative would be.

\section{Conclusion}

Long COVID poses a diagnostic and management challenge to the already stretched secondary healthcare facilities, especially rheumatology. 


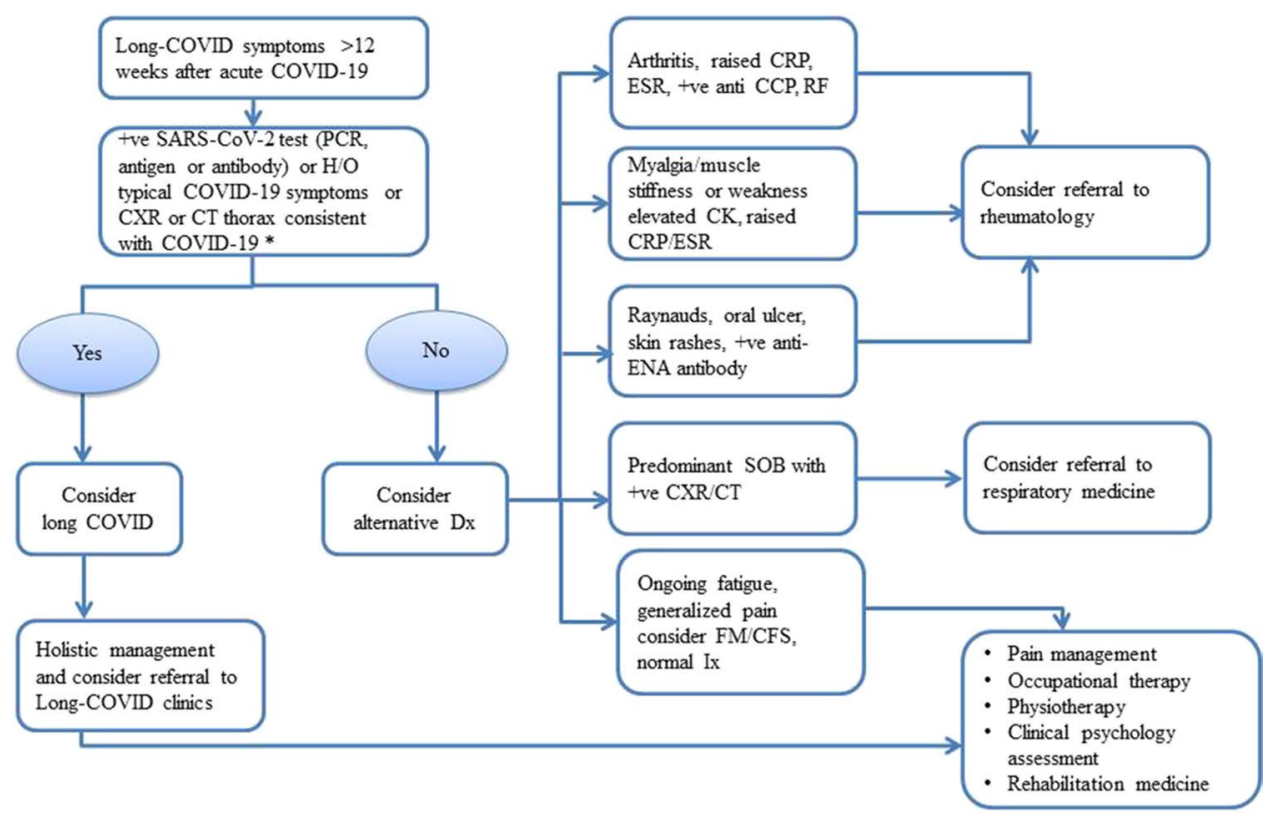

Fig. 2 Flowchart of the management of a patient presenting with long COVID. (anti-CCP anticyclic-citrullinated protein, CFS chronic fatigue syndrome, CK creatinine kinase, CT computed tomography, CXR chest X-ray, Dx diagnosis, ENA extractable nuclear antigen, ESR erythrocyte sedimentation ratio, FM fibromyalgia, H/O history of, Ix investigation, PCR polymerase chain reaction, RF rheumatoid factor, SOB shortness of breath, + ve positive, *in presence of contact with confirmed or suspected COVID-19 within 2 weeks of onset symptoms.)

Despite the success of the mass vaccination programme, the burden of long COVID on healthcare facilities persists. Moreover, due to the multi-organ manifestations associated with its presentation, clinicians such as rheumatologists should be well informed about long COVID. Long COVID, a mimicker of SARD, need to be excluded to ensure a correct diagnosis and management plan is in place.

Author contribution HS and AN were involved in conceptualization, literature search, manuscript writing, editing and referencing. Both authors read and agreed on the final draft submitted.

\section{Declarations}

Disclosures None.

\section{References}

1. Hickie I, Davenport T, Wakefield D et al (2006) Post-infective and chronic fatigue syndromes precipitated by viral and non-viral pathogens: prospective cohort study. BMJ 333(7568):575. https:// doi.org/10.1136/bmj.38933.585764.AE

2. Parums DV (2021) Editorial: long COVID, or post-COVID syndrome, and the global impact on health care. Med Sci Monit 27:e933446. https://doi.org/10.12659/MSM.933446 (Published 2021 Jun 7)
3. ONS 2021 (Prevalence of ongoing symptoms following coronavirus (COVID-19) infection in the UK: 9/09/2021 access. https://www.ons.gov.uk/peoplepopulationandcommunity/healt handsocialcare/conditionsanddiseases/bulletins/prevalence ofongoingsymptomsfollowingcoronaviruscovid19infectionin theuk/2september2021

4. Beeching NJ, Fletcher TE, Beadsworth MBJ (2020) Covid-19: testing times. BMJ. 369:m1403. https://doi.org/10.1136/bmj. m1403 (Published 2020 Apr 8)

5. Moreno-Pérez O, Merino E, Leon-Ramirez JM et al (2021) Post-acute COVID-19 syndrome. Incidence and risk factors: A Mediterranean cohort study. J Infect 82(3):378-383. https://doi. org/10.1016/j.jinf.2021.01.004

6. Mandal S, Barnett J, Brill SE et al (2021) 'Long-COVID': a cross-sectional study of persisting symptoms, biomarker and imaging abnormalities following hospitalisation for COVID19. Thorax 76(4):396-398. https://doi.org/10.1136/thora xjnl-2020-215818

7. Fernández-de-Las-Peñas C, Palacios-Ceña D, Gómez-Mayordomo V, Cuadrado ML, Florencio LL (2021) Defining postCOVID symptoms (post-acute COVID, long COVID, persistent post-COVID): an integrative classification. Int J Environ Res Public Health 18(5):2621. https://doi.org/10.3390/ijerph1805 2621 (Published 2021 Mar 5)

8. Greenhalgh T, Knight M, A'Court C, Buxton M, Husain L (2020) Management of post-acute covid-19 in primary care. BMJ 370:m3026. https://doi.org/10.1136/bmj.m3026 (Published 2020 Aug 11)

9. National Institute for Health and Care Excellence(NICE) (2020) COVID-19 rapid guideline: managing the long-term effects of COVID-19. NICE guideline [NG188] . Published Dec 2020. Available at (https://www.nice.org.uk/guidance/gid-ng10179/ documents/final-scope) accessed 28/12/ 2020 
10. World Health Organisation (WHO) (2021) A clinical case definition of post COVID-19 condition by a Delphi consensus, 6 October 2021. Available at https://www.who.int/publications/i/ item/WHO-2019-nCoV-Post_COVID-19_condition-Clinical_ case_definition-2021.1 Accessed 25/10/2021

11. Mahase E (2020) Covid-19: two antibody tests are "highly specific" but vary in sensitivity, evaluations find. BMJ 369:m2066. https://doi.org/10.1136/bmj.m2066 (Published 2020 May 21)

12. Falahi S, Kenarkoohi A (2020) COVID-19 reinfection: prolonged shedding or true reinfection? New Microbes New Infect 38:100812. https://doi.org/10.1016/j.nmni.2020.100812

13. Ladds E, Rushforth A, Wieringa $S$ et al (2020) Persistent symptoms after Covid-19: qualitative study of 114 "long covid" patients and draft quality principles for services. BMC Health Serv Res 20(1):1144. https://doi.org/10.1186/s12913-020-06001y (Published 2020 Dec 20)

14. Sudre C, Murray B, Varsavsky T, et al (2020) Attributes and predictors of Long-COVID: analysis of COVID cases and their symptoms collected by the Covid Symptoms Study App. medRxiv 10.19.20214494; https://doi.org/10.1101/2020.10.19.20214494

15. Halpin SJ, McIvor C, Whyatt G et al (2021) Postdischarge symptoms and rehabilitation needs in survivors of COVID-19 infection: a cross-sectional evaluation. J Med Virol 93(2):1013-1022. https://doi.org/10.1002/jmv.26368

16. Lopez-Leon S, Wegman-Ostrosky T, Perelman C, et al (2021) More than 50 long-term effects of COVID-19: a systematic review and meta-analysis. Preprint. medRxiv. 2021.01.27.21250617. Published 2021 Jan 30. https://doi.org/10.1101/2021.01.27.21250 617

17. Carfì A, Bernabei R, Landi F (2020) Gemelli against COVID19 post-acute care study group. Persistent symptoms in patients after acute COVID-19. JAMA 324(6):603-605. https://doi.org/ 10.1001/jama.2020.12603

18. Garrigues E, Janvier P, Kherabi Y et al (2020) Post-discharge persistent symptoms and health-related quality of life after hospitalization for COVID-19. J Infect 81(6):e4-e6. https://doi.org/ 10.1016/j.jinf.2020.08.029

19. Huang $\mathrm{C}$, Huang L, Wang $\mathrm{Y}$ et al (2021) 6-month consequences of COVID-19 in patients discharged from hospital: a cohort study. Lancet 397(10270):220-232. https://doi.org/10.1016/S01406736(20)32656-8

20. Goërtz YMJ, Van Herck M, Delbressine JM et al (2020) Persistent symptoms 3 months after a SARS-CoV-2 infection: the postCOVID-19 syndrome? ERJ Open Res 6:00542-02020. https://doi. org/10.1183/23120541.00542-2020

21. Peghin M, Palese A, Venturini M et al (2021) Post-COVID-19 symptoms 6 months after acute infection among hospitalized and non-hospitalized patients [published online ahead of print, 2021 Jun 7]. Clin Microbiol Infect. S1198-743X(21)00281-0. https:// doi.org/10.1016/j.cmi.2021.05.033

22. Sykes DL, Holdsworth L, Jawad N, Gunasekera P, Morice AH, Crooks MG (2021) Post-COVID-19 symptom burden: what is long-COVID and how should we manage it? Lung 199(2):113119. https://doi.org/10.1007/s00408-021-00423-z

23. Augustin M, Schommers P, Stecher M et al (2021) Post-COVID syndrome in non-hospitalised patients with COVID-19: a longitudinal prospective cohort study. Lancet Reg Health Eur 6:100122. https://doi.org/10.1016/j.lanepe.2021.100122

24. Salamanna F, Veronesi F, Martini L, Landini MP, Fini M (2021) Post-COVID-19 syndrome: the persistent symptoms at the postviral stage of the disease. A systematic review of the current data. Front Med (Lausanne) 8:653516. https://doi.org/10.3389/fmed. 2021.653516 (Published 2021 May 4)

25. Vlachoyiannopoulos PG, Magira E, Alexopoulos H et al (2020) Autoantibodies related to systemic autoimmune rheumatic diseases in severely ill patients with COVID-19. Ann Rheum Dis 79:1661-1663

26. Lerma LA, Chaudhary A, Bryan A, Morishima C, Wener MH, Fink SL (2020) Prevalence of autoantibody responses in acute coronavirus disease 2019 (COVID-19). J Transl Autoimmun 3:100073. https://doi.org/10.1016/j.jtauto.2020.100073

27. Litwin CM, Binder SR (2016) ANA testing in the presence of acute and chronic infections. J Immunoassay Immunochem 37(5):439-452. https://doi.org/10.1080/15321819.2016.1174136

28. Derksen VFAM, Kissel T, Lamers-Karnebeek FBG, et al (2021) Onset of rheumatoid arthritis after COVID-19: coincidence or connected? [published online ahead of print, 2021 Mar 1]. Ann Rheum Dis. annrheumdis-2021-219859. https://doi.org/10.1136/ annrheumdis-2021-219859

29. Callow KA, Parry HF, Sergeant M, Tyrrell DA (1990) The time course of the immune response to experimental coronavirus infection of man. Epidemiol Infect 105(2):435-446. https://doi.org/10. 1017/s0950268800048019

30. Raman B, Cassar MP, Tunnicliffe EM et al (2021) Medium-term effects of SARS-CoV-2 infection on multiple vital organs, exercise capacity, cognition, quality of life and mental health, posthospital discharge. EClinicalMedicine 31:100683. https://doi.org/ 10.1016/j.eclinm.2020.100683 (Published 2021 Jan 7)

31. Liao B, Liu Z, Tang L et al (2021) Longitudinal clinical and radiographic evaluation reveals interleukin- 6 as an indicator of persistent pulmonary injury in COVID-19. Int J Med Sci 18(1):29-41. https://doi.org/10.7150/ijms.49728

32. Marvisi M, Ferrozzi F, Balzarini L et al (2020) First report on clinical and radiological features of COVID-19 pneumonitis in a Caucasian population: factors predicting fibrotic evolution. Int J Infect Dis 99:485-488. https://doi.org/10.1016/j.ijid.2020.08.054)

33. Joo YB, Lim YH, Kim KJ, Park KS, Park YJ (2019) Respiratory viral infections and the risk of rheumatoid arthritis. Arthritis Res Ther 21(1):199. https://doi.org/10.1186/s13075-019-1977-9

34. Ono K, Kishimoto M, Shimasaki T et al (2020) Reactive arthritis after COVID-19 infection. RMD Open 6:e001350. https://doi.org/ 10.1136/rmdopen-2020-001350

35. Gasparotto M, Framba V, Piovella C, Doria A, Iaccarino L (2021) Post-COVID-19 arthritis: a case report and literature review. Clin Rheumatol 40(8):3357-3362. https://doi.org/10.1007/ s10067-020-05550-1

36. Hønge BL, Hermansen MF, Storgaard M (2021) Reactive arthritis after COVID-19. BMJ Case Rep 14(3):e241375. https://doi.org/ 10.1136/bcr-2020-241375

37. Petersen MS, Kristiansen MF, Hanusson KD, et al (2020) Long COVID in the Faroe Islands - a longitudinal study among nonhospitalized patients [published online ahead of print, $2020 \mathrm{Nov}$ 30]. Clin Infect Dis. ciaa1792. https://doi.org/10.1093/cid/ciaa1 792

38. Roongta R, Chattopadhyay A, Ghosh A (2021) Correspondence on 'Onset of rheumatoid arthritis after COVID-19: coincidence or connected?' [published online ahead of print, 2021 Apr 27]. Ann Rheum Dis. annrheumdis-2021-220479. https://doi.org/10. 1136/annrheumdis-2021-220479

39. Batur EB, Korez MK, Gezer IA, Levendoglu F, Ural O (2021) Musculoskeletal symptoms and relationship with laboratory findings in patients with COVID-19. Int J Clin Pract 75(6):e14135. https://doi.org/10.1111/ijcp.14135

40. Zhang Q, Shan KS, Minalyan A, O'Sullivan C, Nace T (2020) A rare presentation of coronavirus disease 2019 (COVID-19) induced viral myositis with subsequent rhabdomyolysis. Cureus 12(5):e8074. https://doi.org/10.7759/cureus.8074

41. Beydon M, Chevalier K, Al Tabaa O, et al (2020) Myositis as a manifestation of SARS-CoV-2. Ann Rheum Dis https://doi.org/ 10.1136/annrheumdis-2020-217573. [Epub ahead of print: $23 \mathrm{Apr}$ 2020].pmid:http://www.ncbi.nlm.nih.gov/pubmed/32327427 
42. Zhang H, Charmchi Z, Seidman RJ, et al (2020) COVID-19-associated myositis with severe proximal and bulbar weakness. Muscle Nerve 62:E57-60.https://doi.org/10.1002/mus.27003 pmid:http:// www.ncbi.nlm.nih.gov/pubmed/32535934

43. Chan KH, Farouji I, Abu Hanoud A, Slim J (2020) Weakness and elevated creatinine kinase as the initial presentation of coronavirus disease 2019 (COVID-19). Am J Emerg Med 38(7):1548.e1-1548.e3. https://doi.org/10.1016/j.ajem.2020.05. 015

44. Rivera-Morales MD, Pell R, Rubero J, Ganti L (2020) Acute myopericarditis in the post COVID-19 recovery phase. Cureus. 12(10):e11247. https://doi.org/10.7759/cureus.11247 (Published 2020 Oct 29)

45. Cares-Marambio K, Montenegro-Jiménez Y et al (2021) Prevalence of potential respiratory symptoms in survivors of hospital admission after coronavirus disease 2019 (COVID19): a systematic review and meta-analysis. Chron Respir Dis 18:14799731211002240. https://doi.org/10.1177/1479973121 1002240

46. Wu X, Liu X, Zhou Y et al (2021) 3-month, 6-month, 9-month, and 12 -month respiratory outcomes in patients following COVID-19-related hospitalisation: a prospective study. Lancet Respir Med 9(7):747-754. https://doi.org/10.1016/S22132600(21)00174-0

47. Dani M, Dirksen A, Taraborrelli P et al (2021) Autonomic dysfunction in 'long COVID': rationale, physiology and management strategies. Clinical medicine (London, England) 21(1):e63-e67. https://doi.org/10.7861/clinmed.2020-0896

48. George PM, Barratt SL, Condliffe R et al (2020) Respiratory follow-up of patients with COVID-19 pneumonia. Thorax 75:1009-1016

49. Ojha V, Mani A, Pandey NN, Sharma S, Kumar S (2020) CT in coronavirus disease 2019 (COVID-19): a systematic review of chest CT findings in 4410 adult patients. Eur Radiol 30(11):6129-6138. https://doi.org/10.1007/ s00330-020-06975-7

50. Wang Y, Dong C, Hu Y et al (2020) Temporal changes of CT findings in 90 patients with COVID-19 pneumonia: a longitudinal study. Radiology 296(2):E55-E64. https://doi.org/10.1148/ radiol.2020200843

51. Deng L, Khan A, Zhou W et al (2020) Follow-up study of clinical and chest CT scans in confirmed COVID-19 patients. Radiol Infect Dis 7(3):106-113. https://doi.org/10.1016/j.jrid.2020.07. 002

52. Zhang H, Charmchi Z, Seidman RJ et al (2020) COVID-19-associated myositis with severe proximal and bulbar weakness. Muscle Nerve 62(3):E57-E60. https://doi.org/10.1002/mus. 27003

53. Tabatabaei SMH, Rajebi H, Moghaddas F, Ghasemiadl M, Talari H (2020) Chest CT in COVID-19 pneumonia: what are the findings in mid-term follow-up? Emerg Radiol 27(6):711719. https://doi.org/10.1007/s10140-020-01869-z

54. Mariano RZ, Rio APTD, Reis F (2020) Covid-19 overlapping with systemic sclerosis. Rev Soc Bras Med Trop. 53:e20200450. https://doi.org/10.1590/0037-8682-0450-2020 (Published 2020 Sep 21)

55. Cao M, Zhang S, Chu D et al (2020) COVID-19 or clinical amyopathic dermatomyositis associated rapidly progressive interstitial lung disease? A case report. BMC Pulm Med 20(1):304. https://doi.org/10.1186/s12890-020-01335-z

56. Schwartzberg LN, Advani S, Clancy DC, Lin A, Jorizzo JL (2021) A systematic review of dermatologic manifestations among adult patients with COVID-19 diagnosis. Skin Health Dis 1(2):e20. https://doi.org/10.1002/ski2.20
57. McMahon DE, Gallman AE, Hruza GJ et al (2021) Long COVID in the skin: a registry analysis of COVID-19 dermatological duration. Lancet Infect Dis 21(3):313-314. https://doi. org/10.1016/S1473-3099(20)30986-5

58. Killion L, Beatty PE, Salim A (2021) Rare cutaneous manifestation of COVID-19. BMJ Case Rep. 14(1):e240863. https://doi. org/10.1136/bcr-2020-240863 (Published 2021 Jan 28)

59. Mehta P, Bunker CB, Ciurtin C et al (2021) Chilblain-like acral lesions in long COVID-19: management and implications for understanding microangiopathy. Lancet Infect Dis 21(7):912. https://doi.org/10.1016/S1473-3099(21)00133-X

60. Anders HJ, Sigl T, Schattenkirchner M (2001) Differentiation between primary and secondary Raynaud's phenomenon: a prospective study comparing nailfold capillaroscopy using an ophthalmoscope or stereomicroscope. Ann Rheum Dis 60:407-409

61. Mohabbat AB, Mohabbat NML, Wight EC (2020) Fibromyalgia and chronic fatigue syndrome in the age of COVID-19. Mayo Clin Proc Innov Qual Outcomes 4(6):764-766. https://doi.org/ 10.1016/j.mayocpiqo.2020.08.002

62. Clauw D, Häuser W, Cohen S, Fitzcharles M (2020) Considering the potential for an increase in chronic pain after the COVID-19 pandemic. Pain 161(8):1694-1697. https://doi.org/ 10.1097/j.pain.0000000000001950

63. Wong TL, Weitzer DJ (2021) Long COVID and myalgic encephalomyelitis/chronic fatigue syndrome (ME/CFS)-a systemic review and comparison of clinical presentation and symptomatology. Medicina (Kaunas) 57(5):418. https://doi.org/10.3390/ medicina57050418 (Published 2021 Apr 26)

64. Graham EL, Clark JR, Orban ZS et al (2021) Persistent neurologic symptoms and cognitive dysfunction in non-hospitalized Covid-19 "long haulers." Ann Clin Transl Neurol 8(5):10731085. https://doi.org/10.1002/acn3.51350

65. Ursini F, Ciaffi J, Mancarella L et al (2021) Fibromyalgia: a new facet of the post-COVID-19 syndrome spectrum? Results from a web-based survey. RMD Open 7:e001735. https://doi.org/10. 1136/rmdopen-2021-001735

66. Wood E, Hall KH, Tate W (2021) Role of mitochondria, oxidative stress and the response to antioxidants in myalgic encephalomyelitis/chronic fatigue syndrome: a possible approach to SARS-CoV-2 "long-haulers"? Chronic Dis Transl Med 7(1):1426. https://doi.org/10.1016/j.cdtm.2020.11.002

67. González-Hermosillo JA, Martínez-López JP, Carrillo-Lampón SA et al (2021) Post-acute COVID-19 symptoms, a potential link with myalgic encephalomyelitis/chronic fatigue syndrome: a 6-month survey in a Mexican cohort. Brain Sci 11(6):760. https://doi.org/10.3390/brainsci11060760 (Published 2021 Jun 8)

68. Grech S, Borg JN, Cuschieri S (2021) Back pain: An aftermath of Covid-19 pandemic? A Malta perspective [published online ahead of print, 2021 Jun 6]. Musculoskeletal Care. https://doi. org/10.1002/msc. $1574.10 .1002 / \mathrm{msc} .1574$

69. Liu Y, Sawalha AH, Lu Q (2021) COVID-19 and autoimmune diseases. Curr Opin Rheumatol 33(2):155-162. https://doi.org/ 10.1097/BOR.0000000000000776

70. Vojdani A, Vojdani E, Kharrazian D (2021) Reaction of human monoclonal antibodies to SARS-CoV-2 proteins with tissue antigens: implications for autoimmune diseases. Front Immunol 11:617089. https://doi.org/10.3389/fimmu.2020.617089

71. Raveendran AV, Jayadevan R, Sashidharan S (2021) Long COVID: an overview. Diabetes Metab Syndr 15(3):869-875. https://doi.org/10.1016/j.dsx.2021.04.007)

72. Raj SR, Arnold AC, Barboi A et al (2021) Long-COVID postural tachycardia syndrome: an American Autonomic Society statement. Clin Auton Res 31(3):365-368. https://doi.org/10. 1007/s10286-021-00798-2 
73. Crook H, Raza S, Nowell J, Young M, Edison P (2021) Long covid-mechanisms, risk factors, and management. BMJ (Clinical research ed) 374:n1648. https://doi.org/10.1136/bmj.n1648

74. Watad A, De Marco G, Mahajna H et al (2021) Immune-mediated disease flares or new-onset disease in 27 subjects following mRNA/DNA SARS-CoV-2 vaccination. Vaccines (Basel) 9(5):435. https://doi.org/10.3390/vaccines9050435 (Published 2021 Apr 29)
75. Terracina KA, Tan FK (2021) Flare of rheumatoid arthritis after COVID-19 vaccination. Lancet Rheumatol 3(7):e469-e470. https://doi.org/10.1016/S2665-9913(21)00108-9

76. Nune A, Iyengar KP, Ish P, Varupula B, Musat CA, Sapkota HR (2021) Emergence of new onset SLE following SARS-CoV-2 vaccination, QJM, hcab229, https://doi.org/10.1093/qjmed/hcab229

Publisher's note Springer Nature remains neutral with regard to jurisdictional claims in published maps and institutional affiliations. 